\title{
Almacenes y flujos de carbono en humedales de agua dulce en México
}

\section{Carbon fluxes and stocks in freswater wetlands in Mexico}

\author{
María Elizabeth Hernández ${ }^{*}$ y Patricia Moreno-Casasola Barceló²
}

1 Instituto de Ecología A.C. Red de Manejo Biotecnológico de Recursos. Xalapa, Veracruz, México.

\author{
2 Instituto de Ecología A.C. Red de Ecología Funcional. *Autor de correspondencia. elizabeth.hernandez@inecol.mx \\ Xalapa, Veracruz, México.
}

\section{RESUMEN}

Este trabajo hace una revisión bibliográfica sobre los almacenes y flujos de carbono en humedales de agua dulce en México. Se encontraron datos sobre almacenes de carbono en suelo, biomasa aérea y raíces en humedales herbáceos (popales, tulares y carrizales), humedales arbóreos (selvas inundables y palmares) y en humedales transformados en potreros que aún se inundan, para tres estados del sureste mexicano: Veracruz, Yucatán y Chiapas. El mayor almacén de carbono se ha registrado para el suelo de las selvas inundables (150 Mg C $\mathrm{ha}^{-1}$ a $650 \mathrm{Mg} \mathrm{C} \mathrm{ha}^{-1}$. Los almacenes de carbono en la biomasa área de las selvas inundables fueron de $10 \mathrm{Mg} \mathrm{C} \mathrm{ha}^{-1}$ a $162 \mathrm{Mg} \mathrm{C}^{-1}$, siendo también los valores más altos. Con respecto a los flujos de carbono medidos como caída de hojarasca en selvas inundables, solo se encontraron datos para Veracruz $\left(6 \mathrm{Mg} \mathrm{C} \mathrm{ha}^{-1}\right.$ año $^{-1}$ a $9 \mathrm{Mg} \mathrm{C} \mathrm{ha}^{-1}$ año $\left.{ }^{-1}\right)$. Los flujos de metano y bióxido de carbono estuvieron en el intervalo de $0.01 \mathrm{mg} \mathrm{C} \mathrm{m}^{-2} \mathrm{~d}^{-1}$ a $1244 \mathrm{mg} \mathrm{C} \mathrm{m}^{-2} \mathrm{~d}^{-1} \mathrm{y}^{0.009} \mathrm{~g} \mathrm{C} \mathrm{m}^{-2} \mathrm{~d}^{-1}$ a $11 \mathrm{~g} \mathrm{C} \mathrm{m}^{-2} \mathrm{~d}^{-1}$ respectivamente, para Veracruz y Tabasco, y dichas emisiones aumentan hasta 10 veces en humedales transformados en potreros. Se concluye que aún son muy pocas las publicaciones y pocos los sitios estudiados sobre la dinámica del carbono en humedales de agua dulce, por lo que hay que incrementar la investigación en esta línea, ya que los humedales son importantes sumideros de carbono y es necesaria una legislación que proteja las reservas de carbono en estos ecosistemas.

PALABRAS ClAVE: cambio climático, gases de efecto invernadero, materia orgánica, secuestro de carbono, suelos, sumidero de carbono.

\section{ABSTRACT}

In this study, we made a review about carbon stocks and fluxes in Mexican freshwater wetlands. Data for carbon storage in roots, soil and biomass have been reported for marshes, forested wetlands and flooded grasslands in southeast Mexico: Veracruz, Chiapas and Yucatan. The largest carbon stock in freshwater wetlands is in the soil $\left(150 \mathrm{Mg} \mathrm{C} \mathrm{ha}^{-1} \mathrm{a} 650 \mathrm{Mg} \mathrm{C}^{-1}\right)$ and the forested wetlands showed the highest values. The carbon stock in the biomass ranges from $10 \mathrm{Mg} \mathrm{C} \mathrm{ha}^{-1}$ to $162 \mathrm{Mg} \mathrm{C} \mathrm{ha-1}^{-1}$ and forested wetlands showed the highest values. Carbon fluxes measured as litter fall have been reported for forested wetlands in Veracruz $\left(6 \mathrm{Mg} \mathrm{C}^{-1} \mathrm{year}^{-1}\right.$ a $9 \mathrm{Mg} \mathrm{C}^{-1}$ $\mathrm{ha}^{-1}$ year ${ }^{-1}$ ) only. Methane and carbon dioxide fluxes ranged from $0.01 \mathrm{mg} \mathrm{C} \mathrm{m}^{-2} \mathrm{~d}^{-1}$ to $1244 \mathrm{mg} \mathrm{C} \mathrm{m}^{-2} \mathrm{~d}^{-1}$, and $0.009 \mathrm{~g} \mathrm{C} \mathrm{m}^{-2} \mathrm{~d}^{-1}$ to $11 \mathrm{~g}^{-2}$ $\mathrm{C} \mathrm{m}^{-2} \mathrm{~d}^{-1}$ respectively for Veracruz and Tabasco wetlands. Such carbon fluxes increased up to ten times, when wetlands are transformed to grasslands. It is concluded that the number of publications and study sites on carbon dynamics in Mexican freshwater wetlands is still very small. Therefore, it is necessary to increase the research in this area and enact laws that protect these important carbon sinks.

KEYWORDS: climate change, greenhouse gases, organic matter, carbon sinks, soils, carbon sequestration. 


\section{INTRODUCCIÓN}

La Convención Internacional de Ramsar define a los humedales como: "ecosistemas tanto naturales como artificiales que se hallan permanente o temporalmente inundados, ya sea por aguas dulces, salobres o salinas, estancadas o corrientes, y que incluyen regiones ribereñas, costeras o marinas, que no excedan los $6 m$ de profundidad". Dicha definición es amplia, en congruencia con la vocación de la convención, para incluir a varios cuerpos de agua con fines de conservación. Sin embargo, existe una definición más adecuada desde el punto de vista científico, en la cual se definen a los humedales como: "zonas de transición entre los sistemas acuáticos y terrestres, que presentan inundaciones con agua dulce o salobre de forma temporal o permanente; dicha inundación puede ser sujeta o no a la influencia de mareas". La inundación debe ser lo suficientemente larga para permitir el desarrollo de suelos hídricos y, al menos periódicamente, mantener una vegetación predominante de hidrófitas, es decir, plantas adaptadas a vivir en condiciones de inundación (Mitsch y Gosselink, 2007).

México posee apenas $0.6 \%$ de los humedales de todo el mundo, es decir, aproximadamente 3318500 ha (Olmsted, 1993), de las cuales 1567000 ha corresponden a superficies estuáricas o humedales costeros (ContrerasEspinosa y Warner, 2004) y 1751500 ha a humedales continentales (De la Lanza-Espino y García-Calderón, 1995). La superficie continental y el perímetro litoral de la República Mexicana están cubiertos en $16.8 \%$ por humedales. México ha perdido o degradado $62.1 \%$ de sus humedales (Landgrave y Moreno-Casasola, 2012). A nivel nacional, los humedales se encuentran definidos dentro de la Ley de Aguas Nacionales (Diario Oficial de la Federación, 2013) como:

zonas de transición entre los sistemas acuáticos y terrestres que constituyen áreas de inundación temporal o permanente, sujetas o no a la influencia de mareas, como pantanos, ciénagas y marismas, cuyos límites los constituyen el tipo de vegetación hidrófila de presencia permanente o estacional, las áreas en donde el suelo es predominantemente hídrico; y las áreas lacustres o de suelos permanentemente húmedos por la descarga natural de acuiferos.

Moreno-Casasola, Infante-Mata y López-Rosas (2012) clasificaron a los humedales mexicanos de acuerdo con el tipo de las comunidades vegetales; los humedales herbáceos identificados en el país son: carrizales, popales, juncales y potreros inundables; mientras que los humedales arbóreos incluyen: manglares, selvas y palmares inundables. En este trabajo se excluye del análisis a los manglares por tener una hidrología con influencia marina y, además, ya se ha registrado una síntesis sobre los flujos y almacenes de carbono en este tipo de humedales (Herrera-Silveira et al., 2016).

Los humedales ocupan una pequeña porción de la superficie del planeta (aproximadamente 6\%), pero se ha estimado que ellos contienen $15 \times 10^{14} \mathrm{~kg}$ del carbono mundial (Kayranli, Scholz, Mustaf y Hedmark, 2010). En los humedales existen dos grandes almacenes de carbono: el que está almacenado en la biomasa aérea de árboles o vegetación herbácea (hojas, tallos, troncos y ramas) y en la biomasa subterránea (raíces), así como el carbono de la materia orgánica almacenada en el suelo. Los flujos de carbono en humedales comprenden la caída de hojarasca en humedales arbóreos, la exportación de carbono disuelto en agua y la emisión de gases como bióxido de carbono y metano. La descomposición del carbono orgánico en los humedales es un proceso complejo que envuelve procesos aeróbicos y anaeróbicos. La oxidación de la materia orgánica es lenta bajo condiciones anaerobias, lo que ocasiona una acumulación de materia orgánica en los sedimentos de los humedales, que a su vez depende del balance entre las entradas (materia orgánica autóctona (producida en el humedal) más la materia orgánica alóctona (producida $e x$ situ), menos las salidas de la materia orgánica. Las salidas se deben a la descomposición bajo condiciones de inundación, la erosión y exportación de compuestos solubles y en suspensión. Múltiples reacciones bioquímicas que utilizan carbono se llevan a cabo en los humedales debido a la gran cantidad de materia orgánica, lo que promueve la actividad microbiana. Los procesos microbianos de transformación de carbono son la respira- 
ción en la zona aerobia, la fermentación, la metanogénesis, la reducción de sulfato, hierro y nitrato en las zonas anóxicas y anaerobias (Hernández, 2010). Dichos procesos microbianos determinan los flujos de carbono en el agua y los flujos de carbono a la atmósfera $\left(\mathrm{CO}_{2}\right.$ y $\left.\mathrm{CH}_{4}\right)$.

En México, el estudio de los humedales se ha centrado más en los humedales costeros de agua salobre como los manglares (López-Portillo y Ezcurra, 2002; Moreno, Guerrero, Gutiérrez, Ortiz y Palma, 2002; Moreno-Casasola et al., 2002, Aké-Castillo, Vázquez y López-Portillo, 2006) y más recientemente se han empezado a estudiar los humedales herbáceos y arbóreos de agua dulce (López-Rosas, Moreno-Casasola y Mendelssohn, 2005; Escutia-Lara, Gómez-Romero y Lindig-Cisneros, 2009; Infante-Mata, Moreno-Casasola y Madero-Vega, 2012; Campos-Cascaredo et al., 2011; Marín-Muñiz, Hernández y MorenoCasasola, 2014, 2015; Hernández, Marín-Muñiz y Moreno-Casasola, 2015). En cuanto a la dinámica de carbono, también los manglares han recibido más atención que los humedales de agua dulce. Para los manglares existen datos de almacenes de carbono en biomasa y suelo en las regiones propuestas por la Comisión Nacional para el Conocimiento y Uso de la Biodiversidad (Conabio): Pacífico Norte, Pacífico Centro, Pacífico Sur, Golfo de México y Península de Yucatán (Herrera-Silveria et al., 2016), por lo que existe información para todo el país y para las distintas condiciones climáticas y edafológicas bajo las que se encuentran estos humedales. Por el contrario, para los humedales de agua dulce no existen registros a nivel nacional sobre las reservas de carbono en ellos. Los humedales de agua dulce comprenden un grupo de ecosistemas mucho más biodiversos que los manglares. Los humedales de agua dulce incluyen humedales arbóreos (selvas inundables, bosques riparios) y humedales herbáceos (popales, tulares y espejos de agua con vegetación flotante), con una distribución costera y continental. Este tipo de humedales proveen importantes servicios ambientales de regulación, como el mejoramiento de la calidad del agua y el secuestro de carbono, con un alcance más allá de las zonas costeras, por lo cual que es importante conocer la dinámica del carbono en estos ecosistemas en México.

\section{OBJETIVOS}

El objetivo de este trabajo es presentar el estado del conocimiento sobre la dinámica del carbono (almacenes y flujos) en humedales de agua dulce en México a partir de una revisión de la literatura.

\section{MATERIALES Y MÉTODOS}

Se realizó una búsqueda de bibliografía sobre estudios de almacenes y flujos de carbono en humedales de agua dulce en México. La búsqueda se efectuó en bases de datos de artículos científicos y tesis en español utilizando las palabras clave "carbono" y "humedales", en buscadores como SciELO, base de datos de tesis -Universidad Nacional Autónoma de México, Instituto de Ecología, A. C., Google Académico y publicaciones del Programa Mexicano del Carbono (PMC)-. Así mismo, se realizó una búsqueda de artículos en inglés utilizando las palabras clave "carbon wetlands Mexico" en el metabuscador de Conricyt. Es importante mencionar que de los resultados obtenidos se excluyeron los trabajos relacionados con manglares, ya que el objetivo de este estudio fue centrarse en humedales de agua dulce. Los trabajos se agruparon por entidad federativa y por temas (almacenes y flujos).

\section{Resultados}

Se encontró un total de 269 publicaciones sobre humedales de agua dulce en México, de las cuales solamente 6.6\% correspondieron a estudios sobre almacenes y flujos de carbono en humedales de agua dulce (Fig. 1a). Los trabajos sobre carbono incluyeron en su mayoría artículos con factor de impacto, seguidos por capítulos de libro, artículos arbitrados sin factor de impacto y una tesis (Fig. 1b). La mayoría de los estudios se encontraron en las bases de artículos en inglés, incluidos en el meta buscador de Conricyt (9), seguida por SciELO (2) y las publicaciones de PMC (2) (Fig. 1c).

Las publicaciones encontradas se agruparon por entidad federativa (Fig. 2), encontrándose el mayor número de trabajos sobre almacenes y flujos de carbono en humedales de agua dulce en el estado de Veracruz, mientras que para los estados de Tabasco, Yucatán y 


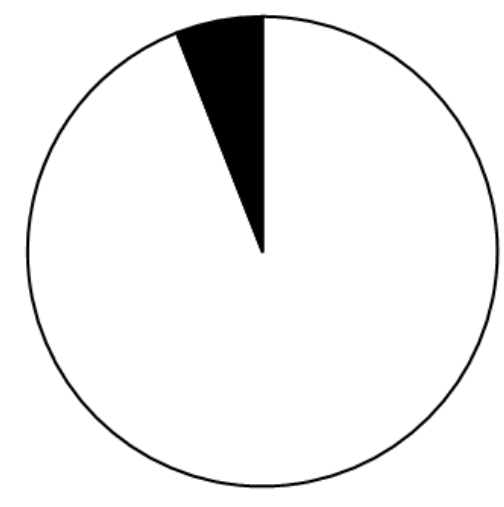

口Otros temas de humedales de agua dulce

- Flujos de carbono en humedales de agua dulce a)

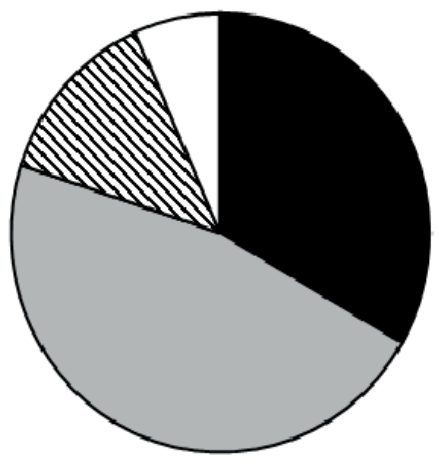

b) $\square$ Articulos con factor de impacto

\$ Articulos sin factor de impacto $\square$ Tesis

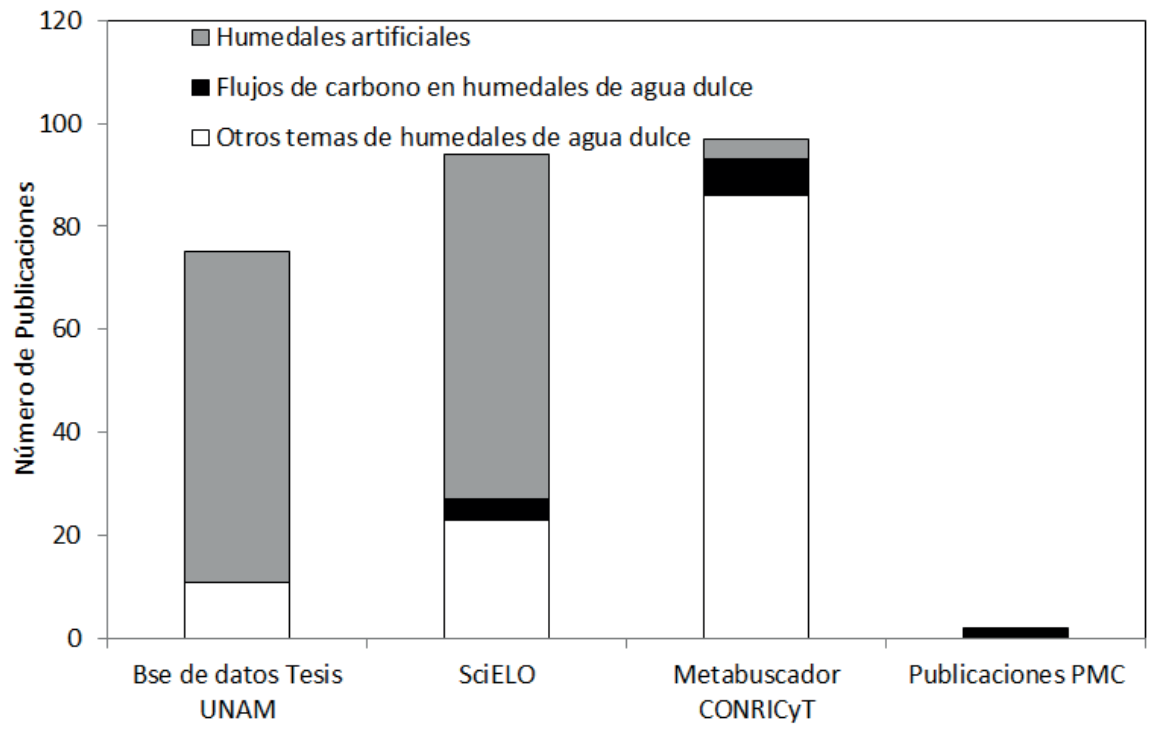

c)

Figura 1. Trabajos publicados sobre almacenes y flujos de carbono en humedales de agua dulce en México.

a) porcentaje del total de trabajos, b) tipo de publicaciones y c) número de trabajos encontrados en distintas bases de datos.

Chiapas solamente se encontró una publicación respectivamente. En el caso de Tabasco fue sobre flujos de carbono y en el caso de Yucatán y Chiapas fueron sobre almacenes de carbono.

El número de publicaciones también se agrupó de acuerdo con el tipo de vegetación de los humedales, encontrándose que para los humedales herbáceos y arbóreos hay un total de ocho y siete publicaciones que abordan el tema, respectivamente, mientras que para potreros inundables hay tres y para palmares inundables hay una sola publicación.

\section{Almacenes de carbono en el suelo}

Los mayores almacenes de carbono en los suelos de humedales de agua dulce mexicanos se han registrado para los humedales arbóreos, también llamados selvas inundables (Fig. 3), siendo mayores en las selvas inundables de Chiapas (610 $\mathrm{Mg} \mathrm{C} \mathrm{ha}^{-1}$ ) que en las selvas inundables de Veracruz (390 Mg C ha-1). En los humedales herbáceos se han encontrado almacenes de carbono en el suelo de $360 \mathrm{Mg} \mathrm{C}^{-1}$ en Veracruz y entre $200 \mathrm{Mg} \mathrm{C}$ ha $^{-1}$ y $250 \mathrm{Mg} \mathrm{C}^{-1}$ en Chiapas y Yucatán. En los potreros inundables de Veracruz, los almacenes de carbono 


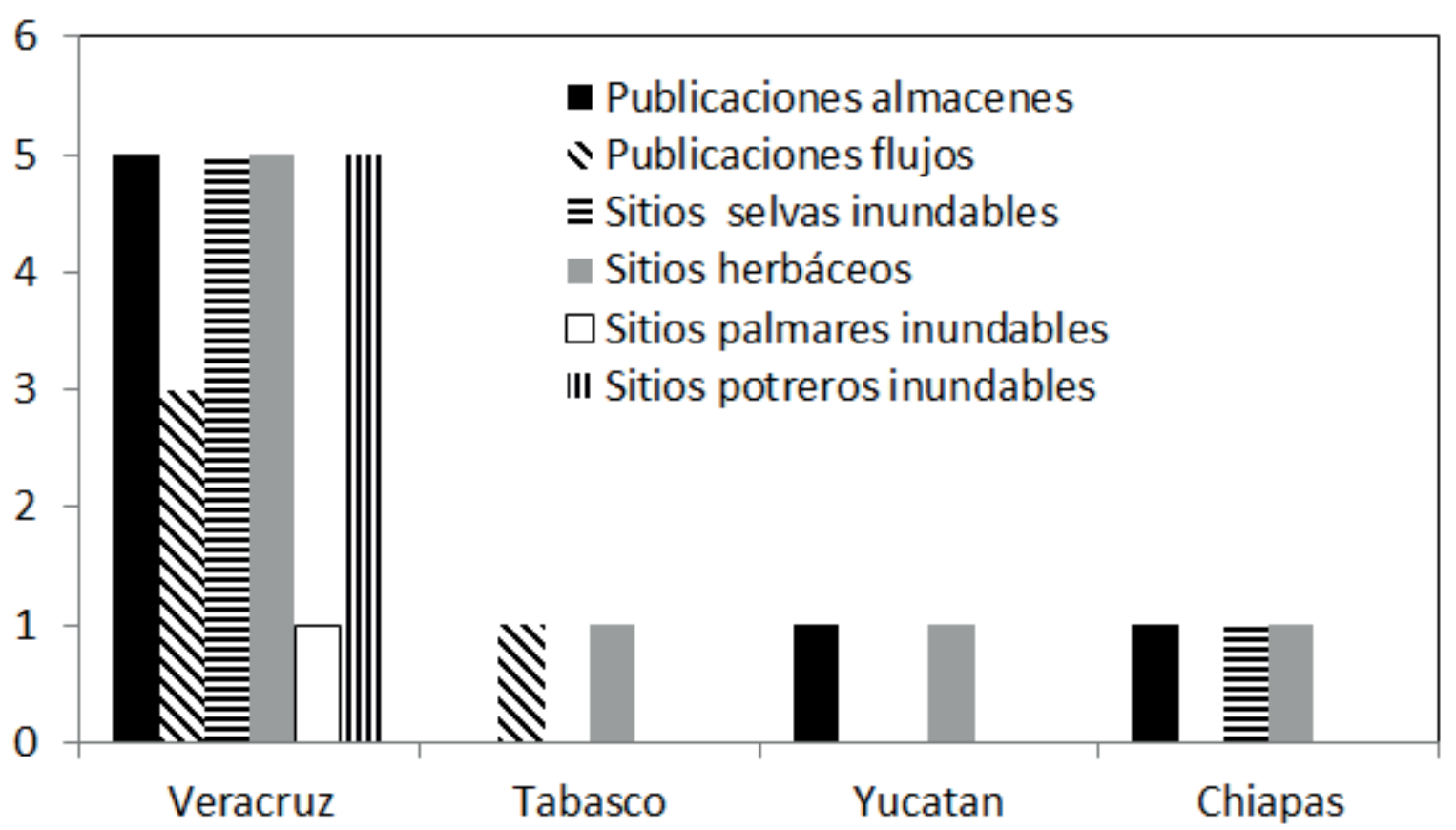

Figura 2. Clasificación de las publicaciones sobre almacenes y flujos de carbono por entidad federativa.

promedio son de $290 \mathrm{Mg} \mathrm{C} \mathrm{ha}^{-1}$, mientras que en los palmares inundables son de $150 \mathrm{Mg} \mathrm{C}^{1}{ }^{1}$ (Adame et al., 2013; Adame et al., 2015; Marín-Muñiz et al., 2014; Hernández, Campos, Marín-Muñiz y Moreno-Casasola, 2016; Moreno-Casasola, Hernández y Campos, 2017).

\section{Almacenes de carbono en la biomasa de raíces}

Para las selvas inundables de Veracruz, se ha encontrado que los almacenes de carbono promedio en raíces son de 2 Mg C ha-1 (Hernández et al., 2016), mientras que para las selvas inundables de Chiapas, los valores registrados son de $18 \mathrm{Mg} \mathrm{C} \mathrm{ha}^{-1}$ (Adame et al., 2015). Para los humedales herbáceos y potreros inundables, solo en Veracruz se tienen datos del carbono almacenado en raíces $\left(11 \mathrm{Mg} \mathrm{C} \mathrm{ha}^{-1}\right.$ y $5 \mathrm{Mg} \mathrm{C} \mathrm{ha-1}^{-1}$.

\section{Almacenes de carbono en biomasa aérea}

El carbono almacenado en la biomasa aérea para las selvas inundables de Chiapas fue de $162 \mathrm{mg} \mathrm{C} \mathrm{ha}^{-1}$, mientras que, para los humedales herbáceos, en Veracruz fue de 4
$\mathrm{Mg} \mathrm{C} \mathrm{ha-1} \mathrm{a} 9 \mathrm{Mg} \mathrm{C} \mathrm{ha}^{-1}$, en Yucatán de $10 \mathrm{Mg} \mathrm{C} \mathrm{ha}^{-1}$ y en Chiapas de $38 \mathrm{Mg} \mathrm{C} \mathrm{ha}^{-1}$ (Adame et al., 2015; Adame et al., 2013; Hernández et al., 2016).

Flujos de carbono a la atmósfera

Existen datos de flujos de $\mathrm{CH}_{4}$ y $\mathrm{CO}_{2}$ para las selvas inundables, humedales herbáceos y porteros inundables en Veracruz (Marín-Muñiz et al., 2015; Hernández et al., 2015). En Tabasco se estudió la emisión de

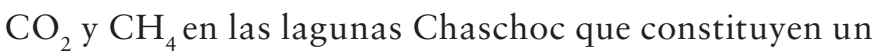
humedal tropical, ribereño al borde del río Usumacinta, es una selva mediana y baja, con actividad agrícola, ganadera y urbana. Las emisiones se midieron en la zona de transición acuático/terrestre (en nivel hidráulico bajo, medio y alto), durante pulsos de inundación (Rojas-Oropeza, Ponce-Mendoza y Cabirol, 2016).

Para Veracruz (Tabla 1) se ha registrado que los mayores flujos de metano ocurrieron en la época de lluvias, sin diferencias entre los diversos humedales, encontrándose los siguientes valores: en las selvas inundables 


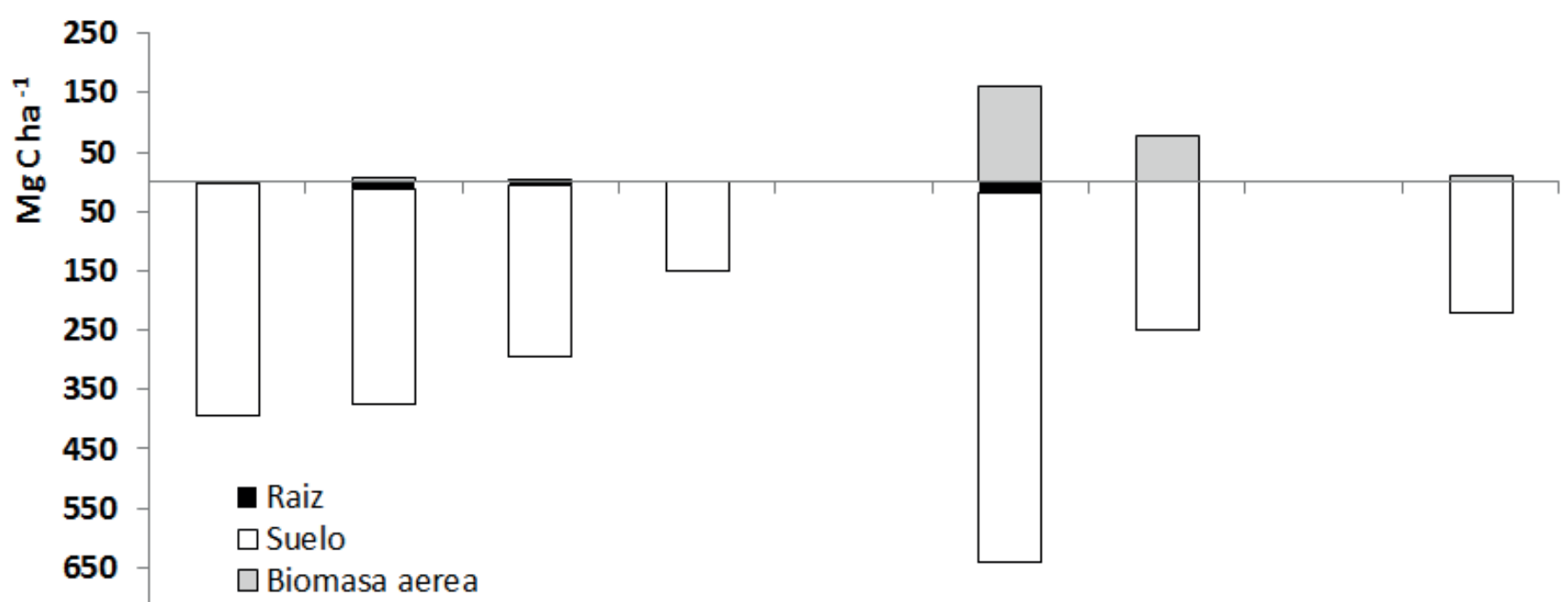

750

Selva I. Herbáceo Potrero I. Palmar I.

Veracruz
Selva I. Herbáceo

Chiapas
Herbáceo

Yucatán

Figura 3. Almacenes de carbono en diferentes tipos de humedales de agua dulce en tres estados del sureste mexicano.

$992 \mathrm{mg} \mathrm{C}-\mathrm{CH}_{4} \mathrm{~m}^{-2} \mathrm{~d}^{-1}$ y en los humedales herbáceos $1244 \mathrm{mg} \mathrm{C}-\mathrm{CH}_{4} \mathrm{~m}^{-2} \mathrm{~d}^{-1}$. Las emisiones de este gas sí fueron significativamente más altas en los potreros inundables (4349 mg C - $\mathrm{CH}_{4} \mathrm{~m}^{-2} \mathrm{~d}^{-1}$ ). En cuanto a las emisiones de $\mathrm{CO}_{2}$, las mayores se observaron en época de secas, sin diferencias entre los humedales arbóreos y herbáceos (10.8 g C - $\mathrm{CO}_{2} \mathrm{~m}^{-2} \mathrm{~d}^{-1}$ y 11 g C $-\mathrm{CO}_{2} \mathrm{~m}^{-2} \mathrm{~d}^{-1}$ ) pero, al igual que el metano, las emisiones de bióxido de carbono fueron más altas en los poteros inundables $(26.38 \mathrm{~g} \mathrm{C}$ $\left.\mathrm{CO}_{2} \mathrm{~m}^{-2} \mathrm{~d}^{-1}\right)$. En Tabasco se observó que las más bajas emisiones de $\mathrm{CO}_{2}$ se registraron en el suelo con un nivel de agua medio $\left(9.2 \mathrm{mg} \mathrm{m}^{-2} \mathrm{~d}^{-1}\right)$ y las mayores en nivel alto (76.57 $\mathrm{mg} \mathrm{m}^{-2} \mathrm{~d}^{-1}$ ). La menor producción de $\mathrm{CH}_{4}$ se presentó en el pulso de transición mínima $\left(0.01 \mathrm{mg} \mathrm{m}^{-2} \mathrm{~d}^{-1}\right)$ y la mayor en el pulso máximo $\left(76.3 \mathrm{mg} \mathrm{m}^{-2} \mathrm{~d}^{-1}\right)$. El suelo ganadero presentó la mayor producción de $\mathrm{CO}_{2}$ durante el pulso de transición mínima y la menor durante el pulso máximo. La menor emisión de $\mathrm{CH}_{4}$ se detectó en el suelo de tránsito urbano y agrícola; la mayor fue en el suelo ganadero.

\section{Flujos de carbono disuelto}

El carbono disuelto en agua ha sido hallado en el intervalo de $25 \mathrm{mg} \mathrm{L}^{-1}$ a $65 \mathrm{mg} \mathrm{L}^{-1}$ para las selvas y humedales herbáceos en Veracruz (Marín-Muñiz et al., 2015). Sin embargo, es importante mencionar que en este estudio no se mencionan los flujos, solo concentraciones; es decir, no se tomaron en cuenta los flujos de agua en los humedales estudiados.

\section{Flujos de carbono en hojarasca}

La productividad medida como flujos de hojarasca en humedales arbóreos de agua dulce, con especies como Pachira acuatica, Ficus spp. y Annona gabra, ha sido registrada para cinco sitios en el Estado de Veracruz, con valores en el intervalo de $9 \mathrm{Mg}$ a $15 \mathrm{Mg}$ (InfanteMata et al., 2012). Sin embargo, en este trabajo no se relaciona dicha productividad con los flujos de carbono. En 2016, también en Veracruz, en humedales de agua dulce arbóreos, se registraron los flujos de carbono a través de la caída de hojarasca anual, en el 
TABla 1. Flujos de carbono como caída de hojarasca y como emisión de gases a la atmósfera en forma de $\mathrm{CH}_{4}$ y $\mathrm{CO}_{2}$ en diferentes tipos de humedales de agua dulce en México.

\begin{tabular}{|c|c|c|c|}
\hline \multirow[t]{3}{*}{ Tipo de Humedal } & \multicolumn{3}{|c|}{ Flujo de carbono } \\
\hline & Como caída de hojarasca & $\mathrm{CH}_{4} \mathrm{mg} \mathrm{m}^{-2} \mathrm{~d}^{-1}$ & $\mathrm{CO}_{2} \mathrm{~g} \mathrm{~m}^{-2} d^{-1}$ \\
\hline & $M g C h a^{-1} a \tilde{n} o^{-1}$ & & \\
\hline Selva inundable Veracruz & $0006-0009$ & $0027-1857$ & $000.5-0015$ \\
\hline Humedal herbáceo Veracruz & NA & $0027-1933$ & $000.5-0018$ \\
\hline Humedal ribereño Tabasco & NA & $00.01-0076$ & $0.009-0.076$ \\
\hline Humedal transformado a potrero Veracruz & NA & $0800-4349$ & $0004-0026$ \\
\hline
\end{tabular}

intervalo de $6 \mathrm{MgC} \mathrm{ha} a^{-1}$ año $^{-1}$ a $9 \mathrm{Mg} \mathrm{C} \mathrm{ha}^{-1}$ año-1 $^{-1}$ (Hernández et al., 2016).

\section{DISCUSIÓN}

La dinámica del carbono en humedales de agua dulce es muy compleja. Involucra la captación de $\mathrm{CO}_{2}$ atmosférico por la vegetación, así como la acumulación de materia orgánica en los suelos debido a la baja tasa de descomposición bajo condiciones de inundación que, a su vez, ocasiona la emisión de metano que es un potente gas de efecto invernadero (Hernández, 2010).

Para el caso de los diferentes tipos de humedales de agua dulce costeros en México, el mayor almacén de carbono se encuentra en el suelo en las selvas y representa hasta 77\% del carbono almacenado (Marín-Muñiz et al., 2014). En los humedales herbáceos puede llegar hasta 98\% del total de carbono almacenado (Marín-Muñiz et al., 2014). Dichas reservas se ven drásticamente impactadas por el cambio de uso de suelo. Los humedales de agua dulce en Veracruz y, en general, en el trópico mexicano están fuertemente amenazados por la actividad ganadera (Moreno-Casasola et al., 2010; Moreno-Casasola, LópezRosas y Rodríguez-Medina, 2014) y gran parte de ellos se han convertido en potreros. Los potreros inundables son humedales perturbados respecto a la vegetación nativa, ya sea porque han sido talados o porque se les han introducido pastos forrajeros para el pastoreo de ganado (LópezRosas et al., 2005). En el Estado de Veracruz, la mayoría de los potreros inundables aún mantienen hidroperiodos y vegetación similares a los de los humedales naturales, como es el caso de los humedales de Alvarado (RodríguezMedina, Moreno-Casasola y Yañez-Arenas, 2017), lo cual ha permitido que no todo el carbono almacenado en el suelo se pierda. Sin embargo, las emisiones de metano y bióxido de carbono en estos humedales transformados son hasta diez veces mayores a las de las selvas inundables (Hernández et al., 2015). Este fenómeno es un foco rojo y deja ver la necesidad de una legislación para la protección y conservación de los humedales de agua dulce, para evitar que se pierdan las reservas de carbono en ellos.

Respecto al carbono almacenado en los suelos de los humedales de agua dulce costeros en Veracruz, Chiapas y Yucatán, aunque difieren entre sí, los valores se encuentran dentro del mismo orden. Sin embargo, para otros almacenes como el carbono almacenado en las raíces, los valores encontrados para las selvas de Chiapas son mucho mayores que para las selvas de Veracruz. Es importante mencionar que, para Chiapas, la cantidad de raíces se calculó mediante ecuaciones alométricas, mientras que, en Veracruz, la cantidad de raíces producidas en un año se calculó mediante la técnica de entierro de cilindros en el suelo con sustratos sin raíces. La diferencia en las metodologías utilizadas hace difícil la comparación; por ello que se deberían establecer protocolos nacionales para la cuantificación de almacenes y flujos de carbono.

Respecto a los flujos de carbono en humedales de agua dulce mexicanos, los datos son aún más escasos, solo hay datos para flujos de gases para dos estados: Tabasco y 
Veracruz. Es importante resaltar que los flujos de $\mathrm{CH}_{4} \mathrm{y}$ $\mathrm{CO}_{2}$ encontrados para Veracruz (Hernández et al., 2015; Marín-Muñiz et al., 2015) están dentro de los intervalos registrados en la literatura (Nahlik y Mitsch, 2011). Sin embargo, comparando los flujos de Veracruz y Tabasco, los flujos de $\mathrm{CH}_{4}$ hallados para Tabasco son 16 veces menores que los correspondientes para Veracruz y los de $\mathrm{CO}_{2}$ hasta 1000 veces menores. En ambos estudios se utilizó el método de la cámara cerrada y la cuantificación de gases por cromatografía de gases, por lo que fueron metodologías similares. Sin embargo, Rojas-Oropeza et al. (2016), no describen detalladamente las fórmulas utilizadas para calcular los flujos, únicamente mencionan que hacen una corrección de la presión atmosférica entre Tabasco y la Ciudad de México para transformar los datos. Las diferencias, entonces, pudieran obedecer a diferentes protocolos de manejo de datos y/o a diferencias en los sitios estudiados, ya que los humedales de Veracruz son sitios que permanecen inundados por al menos nueve meses al año. El humedal muestreado en Tabasco es un humedal ribereño, con inundación de pulso, y en el estudio se muestreó desde zonas que no se inundan hasta zonas cerca del río que reciben la mayor inundación. En una publicación reciente se señalaron flujos de metano en lagunas tropicales rodeadas por manglares en el sureste mexicano (Chuang et al., 2017). Estos autores encontraron valores en el intervalo de $0.036 \mathrm{mg} \mathrm{CH}_{4} \mathrm{~m}^{-2} \mathrm{~d}^{-1}$ a 240 mg $\mathrm{CH}_{4} \mathrm{~m}^{-2} \mathrm{~d}^{-1}$, los cuales son también menores que los registrados para humedales de agua dulce en Veracruz (Marín-Muñiz et al., 2015; Hernández et al., 2015). Sin embargo, los flujos fueron medidos en los espejos de agua de las lagunas y no en los suelos de los manglares, lo cual podría explicar las diferencias. Es necesario contar con líneas de investigación que evalúen la emisión de flujos de GEI en gradientes de humedales costeros con metodologías homogéneas en los mismos tiempos, de tal manera que se pueda generar conocimiento de la dinámica de las emisiones en los diferentes tipos de humedales costeros, incluyendo a los manglares.

Comparando el almacén de carbono total en los humedales de agua dulce con los humedales de agua salobre, como los manglares, y con el carbono almacenado en los ecosistemas terrestres (Tabla 2), se encuentra que los humedales herbáceos de Veracruz y Chiapas almacenan cantidades de carbono similares al promedio nacional de los manglares (Herrera-Silveira et al., 2016) y que las selvas de Chiapas almacenan más del doble; aunque es importante mencionar que los datos de Chiapas corresponden a un solo sitio de estudio y que el valor señalado también está dentro del intervalo nacional para manglares. Para las selvas inundables de Veracruz no hay registros del carbono almacenado en la biomasa aérea, por lo que no se pudo hacer el cálculo del carbono total almacenado. En comparación con el promedio del carbono almacenado en ecosistemas terrestres mexicanos, los humedales de agua dulce pueden almacenar de 4 a 13 veces más que los ecosistemas terrestres. Dicha comparación debe de tomarse con cautela, ya que el área de los humedales de agua dulce es menor que la de los ecosistemas terrestres. Sin embargo, resalta que las reservas de carbono en los humedales de agua dulce son altas y que dichos ecosistemas han sido ignorados en su función como sumideros de carbono a nivel nacional, sobre todo por la falta de un inventario nacional de humedales. En un esfuerzo por mapear los servicios ambientales de los humedales de agua dulce en Veracruz, se han hecho mapas del carbono en algunos humedales de agua dulce (Moreno-Casasola, Monroy-Ibarra, Hernández, Campos y Vázquez-González, 2016). También se ha hecho un esfuerzo aún mayor por resaltar el valor económico de los almacenes de carbono en los suelos de los humedales de agua dulce y manglares del sistema lagunar de Alvarado, Veracruz, para poder traducirlo al lenguaje de los tomadores de decisión y así coadyuvar a la conservación de los servicios ambientales que prestan dichos ecosistemas (Vázquez-González et al., 2017).

Es importante destacar nuevamente que son escasos los datos sobre reservas y flujos de carbono encontrados en humedales de agua dulce en México. Los existentes se refieren únicamente a humedales costeros para tres estados del sureste y, en algunos de ellos, solamente se tiene un sitio estudiado. El estado de Veracruz es el que cuenta 
Tabla 2. Promedio del carbono orgánico total almacenado en diferentes tipos de humedales de agua dulce y su comparación con manglares y con ecosistemas terrestres de acuerdo con la literatura.

\begin{tabular}{ccc}
\hline Ecosistema & $\begin{array}{c}\text { Carbono almacenado } \\
\mathrm{MgC} \mathrm{ha}^{-1}\end{array}$ & $\mathrm{CO}_{2}$ equivalentes ha \\
\hline Ecosistemas terrestres de México * & 062.6 & 0230 \\
Manglares de México ** & 0364 & 1336 \\
Selvas inundables Chiapas & 0802 & 2947 \\
Humedales herbáceos Veracruz & 0381 & 1399 \\
Humedales herbáceos Chiapas & 0337 & 1238 \\
Humedales herbáceos Yucatán & 0241 & 0885 \\
Humedales transformados a potreros Veracruz & 0230 & 0845 \\
\hline
\end{tabular}

*de acuerdo con Vega-López (2008); ** de acuerdo con Herrera-Silveira et al. (2016).

con más sitios de humedales de agua dulce donde se han estudiado los flujos y almacenes de carbono. Resalta que existe una gran variedad de selvas inundables con composiciones de especies muy distintas, así como humedales herbáceos (Moreno-Casasola et al., 2012), por lo que es fundamental incrementar el número de sitios de estudio para abarcar parte de esta variabilidad. Aun cuando existen importantes zonas de humedales continentales en el centro del país (Velazco-Orozco, 2008), no existen trabajos publicados sobre las reservas de carbono en este tipo de humedales. Lo más cercano entre los trabajos publicados es un estudio edafológico de los suelos de los humedales delagodePátzcuaro(Medina-Orozco, García-Calderón, García-Oliva e Ikkonen, 2014), en donde indican un contenido de materia orgánica de $7 \%$ en todo el perfil de $0 \mathrm{~cm}$ - 110 cm; sin embargo, no se calculó el almacén de carbono en el suelo. Aquí se abre una oportunidad de líneas de investigación sobre el carbono almacenado en humedales continentales de México.

\section{CONCLUSIONES}

Los datos de almacenes y flujos de carbono en humedales dulceacuícolas de México son escasos y solo existen datos para humedales de agua dulce costeros en el sureste del país. Comparado con los ecosistemas terres- tres, los humedales de agua dulce pueden almacenar hasta seis veces más carbono. Existe un área de oportunidad de líneas de investigación sobre la dinámica de carbono en humedales de agua dulce costeros y continentales mexicanos. Debido a los escases de datos de flujos de carbono en humedales dulceacuícolas, es pertinente colocar esta demanda en la carpeta de proyectos prioritarios para financiamiento por las diferentes agencias en el país. Las instituciones que cuentan con los equipos para la medición de flujos de gases de carbono deberían hacer esfuerzos para estandarizar las técnicas de medición y capacitar en dichas metodologías estandarizadas, para que se apliquen en más sitios de humedales mexicanos y se puedan obtener datos en las diferentes regiones a nivel nacional.

De acuerdo con los datos existentes, los almacenes de agua dulce costeros pueden almacenar cantidades semejantes a las de los manglares. Sin embargo, los primeros no están protegidos por la legislación. El cambio de uso de suelo de los humedales de agua dulce a pastizales para ganadería ocasiona que disminuyan los almacenes de carbono en ellos y se incremente la emisión de gases de efecto invernadero. Por lo anterior, es urgente incluir a los humedales de agua dulce en la misma ley que protege a los manglares para evitar que se pierdan estos importantes 
sumideros de carbono y poder contribuir a los compromisos mexicanos internacionales en materia de mitigación del cambio climático.

\section{REFERENCIAS}

Adame, M., Kauffman, J., Medina, I., Gamboa, J., Torres, O., Caamal, J., Reza, M., \& Herrera-Silveira, J. (2013). Carbon stock of tropical coastal wetlands within the Karstic landscape of the Mexican Caribbean. PLoS ONE, 8, e56569. doi: 10.1371/journal.pone.0056569.

Adame, M., Santini, N. S., Tovilla, C., Vázquez-Lule, A., Castro, L., \& Guevara, M. (2015). Carbon stocks and soil sequestration rates of tropical riverine wetlands. Biogeosciences, 12, 3805-3818. doi: 10.5194/bg-12-38052015

Aké-Castillo, J. A., Vázquez, G., \& López-Portillo, J. (2006). Litterfall and decomposition of Rhizophora mangle L. in a coastal lagoon in southern Gulf of México. Hydrobiologia, 559, 101-111. doi: 10.1007/s10750-005-0959-x

Campos-Cascaredo, A., Hernández, M. E., Moreno-Casasola, P., Cejudo-Espinosa, E., Robledo, A., \& Infante-Mata, D. M. (2011). Soil water retention and carbon pools in tropical forested wetlands and marshes of the Gulf of Mexico. Hydrological Sciences Journal, 56(8), 1388-1406. doi: 10.1080/02626667.2011.629786

Chuang, P. C., Young, M. B., Dale, A. W., Miller, L. G., Herrera-Silveira, J. A., \& Paytan, A. (2017). Methane fluxes from tropical coastal lagoons surrounded by mangroves, Yucatán, Mexico. Journal of Geophysical Research: Biogeosciences, 122-128 doi: 10.1002/2017JG003761.

Contreras-Espinosa, F., \& B. G. Warner. 2004. Ecosystem characteristics and management considerations from coastal wetlands in Mexico. Hydrobiologia, 511, 233-245. doi: 10.1023/B:HYDR.0000014097.74263.54

De la Lanza-Espino, G., \& García-Calderón, J. L. (comps.). 1995. Lagos y presas de México. México: Centro de Ecología y Desarrollo, A. C.

Escutia-Lara, Y., Gómez-Romero, M., \& Lindig-Cisneros, R. (2009). Nitrogen and phosphorus effect on Typha domingensis Presl. rhizome growth in a matrix of Schoenoplectus ame- ricanus (Pers.) Volkart ex Schinz and Keller Yazmín. Aquatic Botany, 90, 74-77. doi: 10.1016/j.aquabot.2008.06.002

Hernández, M. E. (2010). El papel de los suelos de humedales como sumideros de carbono y fuentes de metano. Terra Latinoamericana, 28(2),139-147.

Hernández, M. E., Marín-Muñiz, J. L., \& Moreno-Casasola, P. (2015). Comparing soil carbon pools and carbon gas fluxes in coastal forested wetlands and flooded grasslands in Veracruz, Mexico. International Journal of Biodiversity Science, Ecosystem Services \& Management, 11, 5-16. doi: 10.1080/21513732.2014.925977

Hernández, M. E., Campos, A., Marín-Muñiz, J. L., \& MorenoCasasola, P. (2016). Almacenes de carbono en selvas inundables, manglares, humedales herbáceos y potreros inundables. En Moreno Casasola, P. (ed.), Servicios ecosistémicos de las selvas y bosques costeros de Veracruz (pp. 121-129). Xalapa, Veracruz, México: Inecol - ITTO - Conafor - INECC

Herrera-Silveira, J., Camacho, R., Pech, Pech, J., Ramírez, R., \& Teutli, H. (2016). Dinámica del carbono (almacenes y flujos) en manglares de México. Terra Latinoamericana $34,61-72$.

Infante-Mata, D. M., Moreno-Casasola, P., \& Madero-Vega, C. (2012). Litterfall of tropical forested wetlands of Veracruz in the coastal floodplains of the Gulf of Mexico. Aquatic Botany, 98(1), 1-11. doi: 10.1016/j.aquabot.2011.11.006

Kayranli, B., Scholz, A., Mustaf, \& Hedmark. (2010). Carbon storage and fluxes within freshwater wetlands: a critical review. Wetlands, 30, 111-124. doi: 10.1007/s13157009-0003-4

López-Rosas, H., Moreno-Casasola, P., \& Mendelssohn, I. A. (2005). Effects of an African grass invasion on vegetation, soil and interstitial water characteristics in a tropical freshwater marsh in La Mancha, Veracruz (Mexico). Journal of Plant Interactions, 1(3), 187-195. doi: 10.1080/17429140600857693

López-Portillo, J., \& Ezcurra, E. (2002). Los manglares de México: Una revisión. Madera y Bosques, número especial, 27-51. doi: 10.21829/myb.2002.801290

Marín-Muñiz, J. L., Hernández, M. E., \& Moreno-Casasola, P. (2014). Comparing soil carbon sequestration in coastal 
freshwater wetlands with various geomorphic features and plant communities in Veracruz, Mexico. Plant Soil, 378, 189-203. doi: 10.1007/s11104-013-2011-7

Marín-Muñiz, J. L., Hernández, M. E., \& Moreno-Casasola, P. (2015). Greenhouse gas emissions from coastal freshwater wetlands in Veracruz Mexico: Effect of plant community and seasonal dynamics. Atmospheric Environment, 107, 107-117. doi: 10.1016/j.atmosenv.2015.02.036

Medina-Orozco, L. E., García-Calderón, N. E., García-Oliva, F., \& Ikkonen, E. (2014). Suelos de humedal del lago de Pátzcuaro, Michoacán, México. Tecnología y Ciencias del Agua, 5(5), 111-124.

Mitsch, W. J., \& J. Gosselink. (2007). Wetlands (4a ed). Nueva York: John Wiley and Sons Inc.

Moreno, E., Guerrero, M. C., Gutiérrez, Ortiz, C., \& Palma, D. J. (2002). Los manglares de Tabasco, una reserva natural de carbono. Madera y Bosques, número especial, 115128. doi: 10.21829/myb.2002.801295

Moreno-Casasola, P., Rojas, J., Zárate, D., Ortiz, M., Lara, A., \& Saavedra, T. 2002. Diagnóstico de los manglares de Veracruz: Distribución, vínculo con los recursos pesqueros y su problemática. Madera y Bosques, número especial, 61-68. doi: 10.21829/myb.2002.801292

Moreno-Casasola, P., Cejudo-Espinosa, E., Capistran-Barradas, A., Infante-Mata, D., Lopez-Rosas, H., CastilloCampos, G., Pale-Pale, J., \& Campos, A. (2010). Floristic composition, diversity and ecology of freshwater marshes in the central coastal plain of Veracruz, Mexico. Boletín de la Sociedad Botánica de México, 87, 29-50.doi: 10.17129/botsci.291

Moreno-Casasola, P. D. M. Infante-Mata y H. López-Rosas. 2012. Tropical freshwater swamps and marshes. In D. P. Batzer, \& A. H. Baldwin (eds.), Wetland habitats of North America: Ecology and conservation concerns (pp. 267-282). University of California Press.

Moreno-Casasola, P., López-Rosas, H., \& Rodríguez-Medina, K. (2014). From tropical wetlands to pastures on the coast of the Gulf of Mexico. Revista Pastos, 42(2), 183-215.

Moreno-Casasola, P., Monroy-Ibarra, R., Hernández, M. E., Campos, A., \& Vázquez-González, C. (2016). La distribución de los servicios ecosistémicos. En P. Moreno Casa- sola (ed.), Servicios Ecosistémicos de las Selvas y Bosques Costeros de Veracruz (pp. 204-276). Xalapa, Veracruz, México: Inecol - ITTO - Conafor - INECC.

Moreno-Casasola, P., Hernández, M. E., \& Campos, A. (2017). Hydrology, soil carbon sequestration and water retention along a coastal wetland gradient in Alvarado Lagoon system, Veracruz Mexico. Journal of Coastal Research, 77, 104-115. doi: 10.2112/SI77-011.1

Nahlik, A. M., \& Mitsch, W. J. (2011). Methane emissions from tropical freshwater wetlands located in different climatic zones of Costa Rica. Global Change Biology, 17, 13211334. doi: 10.1111/j.1365-2486.2010.02190.x

Olmsted, I. (1993). Wetlands of Mexico. In D. F. Whigham, D. Dykyjová, \& S. Hejnÿ (eds.), Wetlands of the world I: Inventory, ecology and management. Handbook of Vegetation Science (pp. 637-678). Dordrcht: Kluwer Academic Publishers.

Rodríguez-Medina, K., Moreno-Casasola, P., \& Yañez-Arenas, C. (2017). Efecto de la ganadería y la variación estacional sobre la composición florística y la biomasa vegetal en los humedales de la costa central del Golfo de México. Acta Botanica, 119, 79-99. doi: 10.21829/ abm119.2017.1233

Rojas-Oropeza, M., Ponce-Mendoza, A., \& Cabirol, N. (2016). Emisión de gases de efecto invernadero y uso de suelo en lagunas de Chastoc (Emiliano Zapata, Tabasco). En F. Paz, \& R. Torres (eds.), Estado actual del conocimiento del ciclo del carbono y sus interacciones en México: Síntesis a 2011 (pp. 267-279). Pachuca, Hidalgo: Programa Mexicano del Carbono, Universidad Autónoma del Estado de Hidalgo e Instituto Nacional de Ecología Texcoco, México.

Vázquez-González, C., Moreno-Casasola, P., Fermán-Almada, J. L., Hernández, M. E., Campos, A., \& Espejel, I. (2017). Mangrove and freshwater wetland conservation through carbon offsets: a cost benefit analysis in the Alvarado Lagoon System Mexico. Environmental Management, 59(2), 274-290. doi:10.1007/s00267-016-0790-3

Vega-López, E. (2008).Valor económico potencial de las Áreas Naturales Protegidas federales de México como sumideros de carbono. The Nature Conservancy-México. 
Velazco-Orozco, J. J. (2008). La ciénega de Chiconahuapan, Estado de México. Un humedal en deterioro constante. Contribuciones desde Coatepec, 15(2), 101-125.
Este documento se debe citar como: Hernández, M. E., \& Moreno-Casasola B., P. (2018). Almacenes y flujos de carbono en humedales de agua dulce en México. Madera y Bosques, 24(Núm. esp.), e2401881. doi: 10.21829/myb.2018.2401881

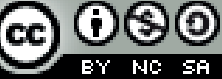

Madera y Bosques por Instituto de Ecología, A.C. se distribuye bajo una Licencia Creative Commons Atribución-No Comercial-Compartir Igual 4.0 Internacional.
Manuscrito recibido el 13 de julio de 2 Aceptado el 19 de febrero de 2018
Acto

Publicado el 29 de octubre de 2018 